International Journal of Pure and Applied Mathematics

Volume 83 No. 1 2013, 193-211

ISSN: 1311-8080 (printed version); ISSN: 1314-3395 (on-line version)

url: http://www.ijpam.eu

doi: http://dx.doi.org/10.12732/ijpam.v83i1.13

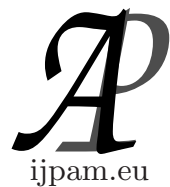

\title{
SCHWARZ-TYPE INTEGRALS IN A BIHARMONIC PLANE
}

\author{
S.V. Gryshchuk ${ }^{1 \S}$, S.A. Plaksa ${ }^{2}$ \\ ${ }^{1,2}$ Institute of Mathematics \\ National Academy of Sciences of Ukraine \\ Tereshchenkivska Str. 3, 01601, Kiev, UKRAINE
}

\begin{abstract}
We consider the commutative algebra $\mathbb{B}$ over the field of complex numbers with the bases $\left\{e_{1}, e_{2}\right\}$ satisfying the conditions $\left(e_{1}^{2}+e_{2}^{2}\right)^{2}=0, e_{1}^{2}+e_{2}^{2} \neq$ 0 . This algebra is unique and it is associated with the biharmonic equation. We consider monogenic functions (having the classic derivative in domains of the biharmonic plane $\left\{x e_{1}+y e_{2}\right\}$, where $x, y$ are real) with values in $\mathbb{B}$. For these functions, we consider a Schwarz-type boundary value problem (associated with the main biharmonic problem) for a half-plane and for a disk of the biharmonic plane.

We obtain solutions in explicit forms by means of Schwarz-type integrals and prove that the mentioned problem is solvable unconditionally for a halfplane but it is solvable for a disk if and only if a certain natural condition is satisfied.
\end{abstract}

AMS Subject Classification: 30G35, 31A30

Key Words: biharmonic equation, biharmonic algebra, biharmonic plane, monogenic function, Schwarz-type boundary value problem, biharmonic Schwarz integral

\section{Introduction}

We say that an associative commutative two-dimensional algebra $\mathbb{B}$ with the

Received: December 12, 2012

(C) 2013 Academic Publications, Ltd.

$\S$ Correspondence author url: www.acadpubl.eu 
unit 1 over the field of complex numbers $\mathbb{C}$ is biharmonic if in $\mathbb{B}$ there exists a biharmonic basis, i.e a bases $\left\{e_{1}, e_{2}\right\}$ satisfying the conditions

$$
\left(e_{1}^{2}+e_{2}^{2}\right)^{2}=0, \quad e_{1}^{2}+e_{2}^{2} \neq 0
$$

V. F. Kovalev and I. P. Mel'nichenko [1] found a multiplication table for a biharmonic basis $\left\{e_{1}, e_{2}\right\}$ :

$$
e_{1}=1, \quad e_{2}^{2}=e_{1}+2 i e_{2},
$$

where $i$ is the imaginary complex unit.

E. Study [2] proved that there exist only two type of two-dimensional algebra with 1 over the field $\mathbb{C}$. In the paper [3] I. P. Mel'nichenko proved that there exists the unique biharmonic algebra $\mathbb{B}$ with a non-biharmonic basis $\{1, \rho\}$ for which

$$
\rho=2 e_{1}+2 i e_{2}
$$

and $\rho^{2}=0$, and he constructed all biharmonic bases in $\mathbb{B}$. It means that only one of algebras considered by E. Study [2] is biharmonic.

Consider a biharmonic plane $\mu:=\left\{\zeta=x e_{1}+y e_{2}: x, y \in \mathbb{R}\right\}$ which is a linear span of the elements $e_{1}, e_{2}$ of the biharmonic basis (2) over the field of real numbers $\mathbb{R}$. With a domain $D$ of the Cartesian plane $x O y$ we associate the congruent $D_{\zeta}:=\left\{\zeta=x e_{1}+y e_{2}:(x, y) \in D\right\}$ in the biharmonic plane $\mu$. In what follows, $\zeta=x e_{1}+y e_{2}$ and $x, y \in \mathbb{R}$.

Inasmuch as divisors of zero don't belong to the biharmonic plane, one can define the derivative $\Phi^{\prime}(\zeta)$ of function $\Phi: D_{\zeta} \longrightarrow \mathbb{B}$ in the same way as in the complex plane:

$$
\Phi^{\prime}(\zeta):=\lim _{h \rightarrow 0, h \in \mu}(\Phi(\zeta+h)-\Phi(\zeta)) h^{-1}
$$

We say that a function $\Phi: D_{\zeta} \longrightarrow \mathbb{B}$ is monogenic in a domain $D_{\zeta}$ if the derivative $\Phi^{\prime}(\zeta)$ exists in every point $\zeta \in D_{\zeta}$.

We use the notion of monogenic function in the sense of existence of derived numbers for this function in the domain $D_{\zeta}$ (cf. $[4,5]$ ).

In the scientific literature the denomination of monogenic function is used else for functions satisfying certain conditions similar to the classical Cauchy Riemann conditions (sf. $[6,7]$ ). Such functions are also called regular functions (sf. [8]) or hyperholomorphic functions (cf. [9, 10]). 
It is established in the paper [1] that a function $\Phi: D_{\zeta} \longrightarrow \mathbb{B}$ is monogenic in a domain $D_{\zeta}$ if and only if the following Cauchy-Riemann condition is satisfied

$$
\frac{\partial \Phi(\zeta)}{\partial y}=\frac{\partial \Phi(\zeta)}{\partial x} e_{2}
$$

It is proved in the paper [1] that a function $\Phi(\zeta)$ having derivatives till fourth order in $D_{\zeta}$ satisfies the two-dimensional biharmonic equation

$$
\left(\Delta_{2}\right)^{2} U(x, y):=\left(\frac{\partial^{4}}{\partial x^{4}}+2 \frac{\partial^{4}}{\partial x^{2} \partial y^{2}}+\frac{\partial^{4}}{\partial y^{4}}\right) U(x, y)=0
$$

in the domain $D$ owing to the relations (1) and

$$
\left(\Delta_{2}\right)^{2} \Phi(\zeta)=\Phi^{(4)}(\zeta)\left(e_{1}^{2}+e_{2}^{2}\right)^{2} .
$$

Therefore, every component $U_{k}: D \longrightarrow \mathbb{R}, k=\overline{1,4}$, of the expansion

$$
\Phi(\zeta)=U_{1}(x, y) e_{1}+U_{2}(x, y) i e_{1}+U_{3}(x, y) e_{2}+U_{4}(x, y) i e_{2}
$$

satisfies also the equation (5), i.e. $U_{k}$ is a biharmonic function in the domain $D$.

It is proved in the paper [11] that a monogenic function $\Phi: D_{\zeta} \longrightarrow \mathbb{B}$ has derivatives $\Phi^{(n)}(\zeta)$ of all orders in the domain $D_{\zeta}$ and, consequently, satisfies the two-dimensional biharmonic equation (5). In the papers $[11,12,13]$ it was also proved such a fact that every biharmomic function $U_{1}(x, y)$ in a bounded simply connected domain $D$ is the first component of the expansion (6) of monogenic function $\Phi: D_{\zeta} \longrightarrow \mathbb{B}$ determined in an explicit form.

Basic analytic properties of monogenic functions in a biharmonic plane are similar to properties of holomorphic functions of the complex variable. More exactly, analogues of the Cauchy integral theorem and integral formula, the Morera theorem, the uniqueness theorem, the Taylor and Laurent expansions are established in papers $[12,14]$.

\section{Statement of Problem}

Consider the following boundary value problem: to find a monogenic function $\Phi: D_{\zeta} \longrightarrow \mathbb{B}$ when values of two components of the expansion (6) are given 
on the boundary $\partial D_{\zeta}$, i.e. the following boundary conditions are satisfied for integer numbers $k$ and $m$ jointed by relation $1 \leq k<m \leq 4$ :

$$
U_{k}(x, y)=u_{k}(\zeta), \quad U_{m}(x, y)=u_{m}(\zeta) \quad \forall \zeta \in \partial D_{\zeta}
$$

where $u_{k}$ and $u_{m}$ are given functions.

This problem was first considered by V.F. Kovalev [15] and was called as the biharmonic Schwarz problem because it is analogous in a certain sense to the classical Schwarz problem on finding an analytic function of complex variable when values of its real part are given on the boundary of domain.

Let us analyse the main ideas of the paper [15]. V.F. Kovalev proved that all biharmonic Schwarz problem are reduced to the main three problems:

1) for $k=1$ and $m=2$ (we shall call it by the (1-2)-problem);

2) for $k=1$ and $m=3$ (we shall call it by the (1-3)-problem);

3) for $k=1$ and $m=4$ (we shall call it by the (1-4)-problem).

Some relations between biharmonic Schwarz problems and problems of the theory of elasticity are described in the paper [15]. In particular, it is shown that the main biharmonic problem (see, for example, [16, p. 202]) on finding a biharmonic function $U: D \longrightarrow \mathbb{R}$ with given limiting values of its partial derivatives $\partial U / \partial x$ and $\partial U / \partial y$ on the boundary $\partial D$ can be reduced to the (1-3)-problem.

If certain natural conditions are satisfied, then the (1-4)-problem is solved in [15] in an explicit form. Further, the (1-2)-problem and the (1-3)-problem are reduced to integro-differential equations with using a conformal mapping of a domain $D_{\zeta}$ onto the half-plane $\Pi^{+}:=\left\{\zeta=x e_{1}+y e_{2}: y>0\right\}$ and the (1-4)-problem as an auxiliary problem. Note that V.F. Kovalev [15] stated only a sketch of solving biharmonic Schwarz problems, and he did not investigate conditions of solvability of these problems.

Having an intention to reduce the (1-3)-problem to integral equations, we study boundary properties of certain integral representations of monogenic functions. Considering the (1-3)-problem for a half-plane and for a disk under natural assumptions on the functions $u_{1}$ and $u_{3}$, we obtain solutions in explicit forms. Moreover, the (1-3)-problem is solvable unconditionally for a half-plane but it is solvable for a disk if and only if a certain condition is satisfied.

\section{Biharmonic Schwarz integral for a half-plane}

Let a function $u: \mathbb{R} \longrightarrow \mathbb{R}$ be continuous and there exists a finite limit

$$
u(\infty):=\lim _{t \rightarrow \infty} u(t) .
$$


Under assumptions that the modulus of continuity

$$
\omega_{\mathbb{R}}(u, \varepsilon)=\sup _{\tau_{1}, \tau_{2} \in \mathbb{R}:\left|\tau_{1}-\tau_{2}\right| \leq \varepsilon}\left|u\left(\tau_{1}\right)-u\left(\tau_{2}\right)\right|
$$

and the local centered (with respect to the infinitely remote point) modulus of continuity

$$
\omega_{\mathbb{R}, \infty}(u, \varepsilon)=\sup _{\tau \in \mathbb{R}:|\tau| \geq 1 / \varepsilon}|u(\tau)-u(\infty)|
$$

of the function $u$ satisfy the Dini conditions

$$
\begin{aligned}
& \int_{0}^{1} \frac{\omega_{\mathbb{R}}(u, \eta)}{\eta} d \eta<\infty, \\
& \int_{0}^{1} \frac{\omega_{\mathbb{R}, \infty}(u, \eta)}{\eta} d \eta<\infty,
\end{aligned}
$$

consider an integral

$$
S_{\Pi^{+}}[u](\zeta):=\frac{1}{\pi i} \int_{-\infty}^{+\infty} \frac{u(t)(1+t \zeta)}{\left(t^{2}+1\right)}(t-\zeta)^{-1} d t \quad \forall \zeta \in \Pi^{+}
$$

that we shall call the biharmonic Schwarz integral for the half-plane $\Pi^{+}$.

Here and in what follows, all integrals along the real axis are understood in the sense of their Cauchy principal values, i.e.

$$
\begin{gathered}
\int_{-\infty}^{+\infty} g(t, \cdot) d t:=\lim _{N \rightarrow+\infty} \int_{-N}^{N} g(t, \cdot) d t, \\
\int_{-\infty}^{+\infty} \frac{g(t, \cdot)}{t-\xi} d t:=\lim _{N \rightarrow+\infty} \lim _{\varepsilon \rightarrow 0+0}\left(\int_{-N}^{\xi-\varepsilon}+\int_{\xi+\varepsilon}^{N}\right) \frac{g(t, \cdot)}{t-\xi} d t, \quad \xi \in \mathbb{R} .
\end{gathered}
$$

The function $S_{\Pi^{+}}[u](\zeta)$ is the principal extension (see [17, p. 165]) into the half-plane $\Pi^{+}$of the complex Schwarz integral

$$
S[u](z):=\frac{1}{\pi i} \int_{-\infty}^{+\infty} \frac{u(t)(1+t z)}{\left(t^{2}+1\right)(t-z)} d t,
$$


which determines a holomorphic function in the half-plane $\{z=x+i y: y>0\}$ of the complex plane $\mathbb{C}$ with the given boundary values $u(t)$ of real part on the real line $\mathbb{R}$. Furthermore, the equality

$$
S_{\Pi^{+}}[u](\zeta)=S[u](z)-\frac{y}{2 \pi} \rho \int_{-\infty}^{\infty} \frac{u(t)}{(t-z)^{2}} d t \quad \forall \zeta=x e_{1}+e_{2} y \in \Pi^{+}
$$

holds, where $z=x+i y$ as well as in what follows.

We use the euclidian norm $\|a\|:=\sqrt{\left|z_{1}\right|^{2}+\left|z_{2}\right|^{2}}$ in the algebra $\mathbb{B}$, where $a=z_{1} e_{1}+z_{2} e_{2}$ and $z_{1}, z_{2} \in \mathbb{C}$.

The following theorem presents sufficient conditions for the existence of boundary values of the biharmonic Schwarz integral on the extended real line $\mathbb{R} \cup\{\infty\}$.

Theorem 1. If a function $u: \mathbb{R} \longrightarrow \mathbb{R}$ has the finite limit (7) and the condition (8) is satisfied, then the equality

$$
\lim _{\zeta \rightarrow \xi, \zeta \in \Pi^{+}} S_{\Pi^{+}}[u](\zeta)=u(\xi)+\frac{1}{\pi i} \int_{-\infty}^{\infty} \frac{u(t)}{t^{2}+1} \frac{1+t \xi}{t-\xi} d t \quad \forall \xi \in \mathbb{R}
$$

is fulfilled. If, in addition, the function $u$ satisfies the condition (9), then

$$
\lim _{\|\zeta\| \rightarrow \infty, \zeta \in \Pi^{+}} S_{\Pi^{+}}[u](\zeta)=u(\infty)-\frac{1}{\pi i} \int_{-\infty}^{\infty} u(t) \frac{t}{t^{2}+1} d t
$$

Proof. In order to prove the equality (11) we use the expression (10) of the biharmonic Schwarz integral. The second summand in the right-hand part of equality (10) tends to zero with $\zeta \rightarrow \xi \in \mathbb{R}$. This statement follows from the equalities

$$
\begin{gathered}
y \int_{-\infty}^{\infty} \frac{u(t)}{(t-z)^{2}} d t=y \int_{-\infty}^{\infty} \frac{u(t)-u(x)}{(t-z)^{2}} d t=y \int_{x-2|y|}^{x+2|y|} \frac{u(t)-u(x)}{(t-z)^{2}} d t+ \\
+y\left(\int_{-\infty}^{x-2|y|}+\int_{x+2|y|}^{\infty}\right) \frac{u(t)-u(x)}{(t-z)^{2}} d t=: I_{1}+I_{2}
\end{gathered}
$$


and the relations

$$
\begin{gathered}
\left|I_{1}\right| \leq|y| \int_{x-2|y|}^{x+2|y|} \frac{|u(t)-u(x)|}{y^{2}} d t \leq 4 \omega_{\mathbb{R}}(u, 2|y|) \rightarrow 0, \quad z \rightarrow \xi \\
\left|I_{2}\right| \leq|y|\left(\int_{-\infty}^{x-2|y|}+\int_{x+2|y|}^{\infty}\right) \frac{|u(t)-u(x)|}{|t-x|^{2}} d t \leq \\
\leq 2|y| \int_{2|y|}^{\infty} \frac{\omega_{\mathbb{R}}(u, \eta)}{\eta^{2}} d \eta \rightarrow 0, \quad z \rightarrow \xi
\end{gathered}
$$

By virtue of the condition (8), the Schwarz integral $S[u](z)$ has limiting values on the real line (it follows, for example, from an appropriate result of the paper [18] for the Cauchy type integral), hence

$$
S[u](z) \rightarrow u(\xi)+\frac{1}{\pi i} \int_{-\infty}^{\infty} \frac{u(t)}{t^{2}+1} \frac{1+t \xi}{t-\xi} d t, z=x+i y \rightarrow \xi \in \mathbb{R}, y>0
$$

Thus, the equality (11) is proved.

In order to prove the equality (12) with using the change of variables $t=$ $-1 / t_{1}, z=-1 / z_{1}$ (see, for example, [19, p. 36]) we rewrite the relation (10) in the form

$$
S_{\Pi^{+}}[u](\zeta)=S[v]\left(z_{1}\right)-\frac{1}{2 \pi} \frac{z_{1}}{\bar{z}_{1}} \rho \operatorname{Im} z_{1} \int_{-\infty}^{\infty} \frac{v\left(t_{1}\right)}{\left(t_{1}-z_{1}\right)^{2}} d t_{1}
$$

where $v\left(t_{1}\right):=u(-1 / t)$ and $\bar{z}_{1}:=\operatorname{Re} z_{1}-i \operatorname{Im} z_{1}$. By virtue of Lemma 1 of the paper [20], the function $v$ satisfies a condition of the form (8). Therefore, the equality (12) can be obtained from the equality (16) by passing to the limit when $z_{1}$ $\rightarrow$ 0 $\operatorname{Im} z_{1}>0$, by analogy with the proof of the equality (11). 


\section{4. (1-3)-problem for a half-plane}

Let continuous functions $u_{1}: \mathbb{R} \longrightarrow \mathbb{R}$ and $u_{3}: \mathbb{R} \longrightarrow \mathbb{R}$ have finite limits of the form (7).

Consider the following (1-3)-problem for the half-plane $\Pi^{+}$: to find a continuous function $\Phi: \overline{\Pi^{+}} \longrightarrow \mathbb{B}$ which is monogenic in the domain $\Pi^{+}$and has the limit

$$
\lim _{\|\zeta\| \rightarrow \infty, \zeta \in \Pi^{+}} \Phi(\zeta)=: \Phi(\infty) \in \mathbb{B},
$$

when values of the components $U_{1}$ and $U_{3}$ of the expansion (6) are given on the real line $\mathbb{R}$, i.e. the following boundary conditions are satisfied:

$$
U_{1}(\xi, 0)=u_{1}(\xi), \quad U_{3}(\xi, 0)=u_{3}(\xi) \quad \forall \xi \in \mathbb{R} .
$$

It follows from Theorem 1 that the function

$$
\Phi(\zeta)=S_{\Pi^{+}}\left[u_{1}\right](\zeta) e_{1}+S_{\Pi^{+}}\left[u_{3}\right](\zeta) e_{2}
$$

is a solution of (1-3)-problem for the half-plane $\Pi^{+}$if the functions $u_{1}$ and $u_{3}$ satisfy the same conditions as the function $u$ in Theorem 1 .

To describe all solutions of (1-3)-problem for $\Pi^{+}$, first consider the homogeneous (1-3)-problem.

Theorem 2. All solutions $\Phi: \overline{\Pi^{+}} \longrightarrow \mathbb{B}$ of the homogeneous (1-3)-problem for the half-plane $\Pi^{+}$with zero data $u_{1}=u_{3} \equiv 0$ are expressed in the form

$$
\Phi(\zeta)=a_{1} i e_{1}+a_{2} i e_{2},
$$

where $a_{1}, a_{2}$ are any real constants.

Proof. We use an expression of monogenic function $\Phi$ via two holomorphic functions $F$ and $F_{0}$ (see, for example, [11]) in the form

$$
\Phi(\zeta)=F(z) e_{1}-\left(\frac{i y}{2} F^{\prime}(z)-F_{0}(z)\right) \rho \quad \forall \zeta \in \Pi^{+} .
$$

Inasmuch as the function $F$ is holomorphic in the half-plane $\{z=x+i y$ : $y>0\}$ and continuous on the closed half-plane $\{z=x+i y: y \geq 0\}$ and have the limit

$$
\lim _{z \rightarrow \infty, \operatorname{Re} z>0} F(z)=: F(\infty)
$$


the following equalities hold:

$$
\begin{gathered}
F(z)=\frac{1}{2 \pi i} \int_{-\infty}^{\infty} \frac{F(t)}{t-z} d t+\frac{F(\infty)}{2}, \\
F^{\prime}(z)=\frac{1}{2 \pi i} \int_{-\infty}^{\infty} \frac{F(t)}{(t-z)^{2}} d t \quad \forall z \in \mathbb{C}: \operatorname{Re} z>0 .
\end{gathered}
$$

Therefore, we obtain the equality

$$
\lim _{z \rightarrow \xi, y>0} y F^{\prime}(z)=0, \quad \forall \xi \in \mathbb{R}
$$

as the result of relations $(13)-(15)$.

In the same way with using the change of variables $t=-1 / t_{1}, z=-1 / z_{1}$ in the integral (20) we obtain the equality

$$
\lim _{z \rightarrow \infty, y>0} y F^{\prime}(z)=0 .
$$

Thus, taking into account the relation (3), we have

$$
\lim _{\zeta \rightarrow \xi, \zeta \in \Pi^{+}} \Phi(\zeta)=\left(F(\xi)+2 F_{0}(\xi)\right) e_{1}+2 i F_{0}(\xi) e_{2} \quad \forall \xi \in \mathbb{R},
$$

and the homogeneous (1-3)-problem for $\Pi^{+}$is reduced to finding holomorphic functions $F, F_{0}$ by solving two classical Schwarz problem for the half-plane $\{z=x+i y: y>0\}$ with the following boundary conditions:

$$
\operatorname{Re}\left(F(\xi)+2 F_{0}(\xi)\right)=0, \quad \operatorname{Re}\left(2 i F_{0}(\xi)\right)=0 \quad \forall \xi \in \mathbb{R} .
$$

In such a way we obtain $F_{0}(z) \equiv a_{2} / 2$ and $F(z) \equiv-a_{2}+i a_{1}$, where $a_{1}, a_{2}$ are any real constants.

Finally, substituting the obtained functions $F, F_{0}$ into the equality (19) and taking into account the relation (3), we obtain the equality (18).

In the following theorem we establish the formula of solutions of the (1-3)problem for the half-plane $\Pi^{+}$.

Theorem 3. Let the functions $u_{1}: \mathbb{R} \longrightarrow \mathbb{R}$ and $u_{3}: \mathbb{R} \longrightarrow \mathbb{R}$ have finite limits of the form (7) and satisfy conditions of the form (8) and (9). Then the general solution of (1-3)-problem for $\Pi^{+}$is expressed in the form

$$
\Phi(\zeta)=S_{\Pi^{+}}\left[u_{1}\right](\zeta) e_{1}+S_{\Pi^{+}}\left[u_{3}\right](\zeta) e_{2}+a_{1} i e_{1}+a_{2} i e_{2},
$$

where $a_{1}, a_{2}$ are any real constants. 
Proof. It is obvious that the formula (21) represents the solution of the (1-3)-problem for $\Pi^{+}$as the sum of particular solution (17) and the general solution (18) of the homogeneous (1-3)-problem.

\section{A biharmonic analogue of Schwarz integral for a disk}

In what follows, $D_{\zeta}:=\left\{\zeta=x e_{1}+y e_{2}:\|\zeta\| \leq 1\right\}$ is the unit disk in the biharmonic plane $\mu$ and $D:=\{z=x+i y:|z| \leq 1\}$ is the unit disk in the complex plane $\mathbb{C}$.

For a continuous function $u: \partial D_{\zeta} \longrightarrow \mathbb{R}$, by $\widehat{u}$ we denote the function defined on the unit circle $\partial D$ of the complex plane $\mathbb{C}$ by the equality $\widehat{u}(z)=u(\zeta)$ for all $z \in \partial D$.

Consider the integral

$$
S_{D_{\zeta}}[u](\zeta):=\frac{1}{2 \pi i} \int_{\partial D_{\zeta}} u(\tau)(\tau+\zeta)(\tau-\zeta)^{-1} \tau^{-1} d \tau \quad \forall \zeta \in D_{\zeta}
$$

that is an analogue of the complex Schwarz integral

$$
\frac{1}{2 \pi i} \int_{\partial D} \widehat{u}(t) \frac{t+z}{t-z} \frac{d t}{t} \quad \forall z \in D
$$

which determines a holomorphic function in the disk $D$ with the given boundary values $\widehat{u}(t)$ of real part on the circle $\partial D$.

Consider also singular integrals which are understood in the sense of their Cauchy principal values, i.e.

$$
S_{\partial D_{\zeta}}[u](\zeta):=\frac{1}{2 \pi i} \lim _{\varepsilon \rightarrow 0+0} \int_{\left\{\tau \in \partial D_{\zeta}:\|\tau-\zeta\| \geq \varepsilon\right\}} u(\tau)(\tau+\zeta)(\tau-\zeta)^{-1} \tau^{-1} d \tau
$$

where $\zeta \in \partial D_{\zeta}$,

$$
S_{0}[\widehat{u}](z):=\frac{1}{2 \pi i} \lim _{\varepsilon \rightarrow 0+0} \int_{\{t \in \partial D:|t-z| \geq \varepsilon\}} \widehat{u}(t) \frac{t+z}{t-z} \frac{d t}{t} \quad \forall z \in \partial D .
$$

It can be proved in a similar way as in the complex plane (see, for example, $[18,19])$ that if the modulus of continuity

$$
\omega(u, \varepsilon)=\sup _{\tau_{1}, \tau_{2} \in \partial D_{\zeta}:\left\|\tau_{1}-\tau_{2}\right\| \leq \varepsilon}\left\|u\left(\tau_{1}\right)-u\left(\tau_{2}\right)\right\|
$$


of the function $u: \partial D_{\zeta} \longrightarrow \mathbb{R}$ satisfies the Dini condition

$$
\int_{0}^{1} \frac{\omega(u, \eta)}{\eta} d \eta<\infty
$$

then the integral (22) has limiting values on $\partial D_{\zeta}$ which are expressed by the formula

$$
\lim _{\xi \rightarrow \zeta, \xi \in D_{\zeta}} S_{D_{\zeta}}[u](\xi)=u(\zeta) e_{1}+S_{\partial D_{\zeta}}[u](\zeta) \quad \forall \zeta \in \partial D_{\zeta} .
$$

Theorem 4. If the function $u: \partial D_{\zeta} \longrightarrow \mathbb{R}$ satisfies the condition (24), then the following equality holds:

$$
\begin{aligned}
& S_{\partial D_{\zeta}}[u](\zeta)=S_{0}[\widehat{u}](z) e_{1}-\left(\frac{y}{2 \pi} \int_{\partial D} \frac{\widehat{u}(t)}{t^{2}} d t\right)^{\left(e_{1}+i e_{2}\right)+} \\
& +\left(\frac{x}{2 \pi} \int_{\partial D} \frac{\widehat{u}(t)}{t^{2}} d t+\frac{1}{2 \pi} \int_{\partial D} \frac{\widehat{u}(t)}{t^{3}} d t \int^{\left(e_{2}-i e_{1}\right)} \quad \forall \zeta \in \partial D_{\zeta} .\right.
\end{aligned}
$$

Proof. Let $\zeta \in \partial D_{\zeta}, \tau:=t_{1} e_{1}+t_{2} e_{2}$ and $t:=t_{1}+i t_{2}$, where $t_{1}, t_{2} \in \mathbb{R}$. We have the equalities $\zeta=z e_{1}-\frac{i y}{2} \rho, \tau=t e_{1}-\frac{i t_{2}}{2} \rho$ and $d \tau=e_{1} d t-\frac{i}{2} \rho d t_{2}$ for $\tau \in \partial D_{\zeta}$. Taking also into account the equalities (see [11])

$$
(\tau-\zeta)^{-1}=\frac{1}{t-z} e_{1}+\frac{i\left(t_{2}-y\right)}{(t-z)^{2}} \rho, \quad \tau^{-1}=\frac{1}{t} e_{1}+\frac{i t_{2}}{t^{2}} \rho,
$$

we obtain the following equality:

$$
\begin{gathered}
S_{\partial D_{\zeta}}[u](\zeta)=S_{0}[\widehat{u}](z) e_{1}+\frac{1}{2 \pi i} \int_{\partial D} \frac{\widehat{u}(t)\left(x t_{2}-t_{1} y\right)}{t^{2}(t-z)} d t\left(\frac{i \rho}{2}\right)+ \\
+\frac{1}{2 \pi i} \int_{\partial D} \frac{\widehat{u}(t)(t+z)\left(\left(t_{2}-y\right) d t_{1}-\left(t_{1}-x\right) d t_{2}\right)}{t(t-z)^{2}}\left(\frac{i \rho}{2}\right)=: \\
=: S_{0}[\widehat{u}](z) e_{1}+\frac{i \rho}{2} I_{3}+\frac{i \rho}{2} I_{4} .
\end{gathered}
$$

Transforming the integrals $I_{3}$ and $I_{4}$ with using the change of variables $t=\exp (i \theta), z=\exp \left(i \theta_{z}\right)$, we obtain the equalities

$$
I_{3}=I_{4}=\frac{-x+i y}{4 \pi} \int_{\partial D} \frac{\widehat{u}(t)}{t^{2}} d t-\frac{1}{4 \pi} \int_{\partial D} \frac{\widehat{u}(t)}{t^{3}} d t .
$$


Substituting obtained expressions for $I_{3}$ and $I_{4}$ into the equality (27) and taking into account the equality $i \rho / 2=i e_{1}-e_{2}$ which follows from the equality (3), we obtain the equality (26).

\section{6. (1-3)-problem for a disk}

Let the functions $u_{1}: \partial D_{\zeta} \longrightarrow \mathbb{R}$ and $u_{3}: \partial D_{\zeta} \longrightarrow \mathbb{R}$ satisfy the condition of the form (24).

Consider the following (1-3)-problem for the unit disk $D_{\zeta}$ : to find a continuous function $\Phi: \overline{D_{\zeta}} \longrightarrow \mathbb{B}$ which is monogenic in the disk $D_{\zeta}$ when values of the components $U_{1}$ and $U_{3}$ of the expansion (6) are given on the circle $\partial D_{\zeta}$, i.e. the following boundary conditions are satisfied:

$$
U_{1}(x, y)=u_{1}(\zeta), \quad U_{3}(x, y)=u_{3}(\zeta) \quad \forall \zeta \in \partial D_{\zeta}
$$

It follows from a biharmonic analogue of the Cauchy integral formula (see Theorem 4 from the paper [14]) that the solution $\Phi(\zeta)$ of (1-3)-problem for $D_{\zeta}$ can be represented as a biharmonic Cauchy integral and, consequently, it can also be represented in the form

$$
\Phi(\zeta)=\frac{1}{2 \pi i} \int_{\partial D_{\zeta}} \varphi(\tau)(\tau+\zeta)(\tau-\zeta)^{-1} \tau^{-1} d \tau \quad \forall \zeta \in D_{\zeta},
$$

where $\varphi: \partial D_{\zeta} \longrightarrow \mathbb{B}$ is a certain continuous function.

We shall find solutions of (1-3)-problem for $D_{\zeta}$ in the class $\mathcal{M}$ of functions represented in the form (29), where the function $\varphi: \partial D_{\zeta} \longrightarrow \mathbb{B}$ satisfies the condition of the form (24). The solvability of the (1-3)-problem for $D_{\zeta}$ in the class $\mathcal{M}$ is described in the following theorem.

Theorem 5. Let the functions $u_{1}: \partial D_{\zeta} \longrightarrow \mathbb{R}$ and $u_{3}: \partial D_{\zeta} \longrightarrow \mathbb{R}$ satisfy the condition of the form (24). Then (1-3)-problem for $D_{\zeta}$ is solvable in the class $\mathcal{M}$ if and only if the following condition is satisfied:

$$
\int_{\partial D_{\zeta}} u_{1}(\zeta) d x+u_{3}(\zeta) d y=0
$$

The general solution is expressed in the form

$$
\Phi(\zeta)=S_{D_{\zeta}}\left[u_{1}\right](\zeta) e_{1}+S_{D_{\zeta}}\left[u_{3}\right](\zeta) e_{2}+
$$




$$
+b \zeta+b_{1} e_{1}+b_{2} e_{2}+i\left(a \zeta+a_{1} e_{1}+a_{2} e_{2}\right),
$$

where

$$
\begin{aligned}
b & :=-\frac{1}{2 \pi} \operatorname{Re} \int_{\partial D} \frac{\widehat{u}_{3}(t)}{t^{2}} d t-\frac{1}{2 \pi} \operatorname{Im} \int_{\partial D} \frac{\widehat{u}_{1}(t)}{t^{2}} d t, \\
b_{1} & :=-\frac{1}{2 \pi} \operatorname{Re} \int_{\partial D} \frac{\widehat{u}_{3}(t)}{t^{3}} d t-\frac{1}{2 \pi} \operatorname{Im} \int_{\partial D} \frac{\widehat{u}_{1}(t)}{t^{3}} d t, \\
b_{2} & :=\frac{1}{2 \pi} \operatorname{Im} \int_{\partial D} \frac{\widehat{u}_{3}(t)}{t^{3}} d t-\frac{1}{2 \pi} \operatorname{Re} \int_{\partial D} \frac{\widehat{u}_{1}(t)}{t^{3}} d t,
\end{aligned}
$$

and $a, a_{1}, a_{2}$ are any real constants.

Proof. Let $\Phi \in \mathcal{M}$ be a solution of the (1-3)-problem for $D_{\zeta}$. Taking into account the equalities (25) and (26), we make a conclusion that the limiting values of the function $\Phi$ on the boundary $\partial D_{\zeta}$ can be represented in the form

$$
\Phi(\zeta)=F_{1}(z) e_{1}+F_{2}(z) e_{2}+c_{1} x+c_{2} y+c_{3} \quad \forall \zeta \in \partial D_{\zeta},
$$

where $F_{1}: \bar{D} \longrightarrow \mathbb{C}, F_{2}: \bar{D} \longrightarrow \mathbb{C}$ are certain continuous functions holomorphic in the disk $D$, and $c_{1}, c_{2}, c_{3} \in \mathbb{B}$. Moreover, the functions $F_{1}, F_{2}$ can be represented by complex Schwarz integrals of the form (23). Therefore, the equality (32) can be rewritten as

$$
\begin{gathered}
\Phi(\zeta)=g_{1}(\zeta) e_{1}+S_{\partial D_{\zeta}}\left[g_{1}\right](\zeta)+ \\
+e_{2}\left(g_{3}(\zeta) e_{1}+S_{\partial D_{\zeta}}\left[g_{3}\right](\zeta)\right)+h_{1} x+h_{2} y+h_{3} \quad \forall \zeta \in \partial D_{\zeta},
\end{gathered}
$$

where $g_{1}: \partial D_{\zeta} \longrightarrow \mathbb{R}, \quad g_{3}: \partial D_{\zeta} \longrightarrow \mathbb{R}$ are certain functions satisfying the condition of the form (24), and $h_{1}, h_{2}, h_{3} \in \mathbb{B}$.

It follows from a biharmonic analogue of the Cauchy integral theorem (see Theorem 4 from the paper [14]) that for the function (33) the following equalities must be fulfilled:

$$
\int_{\partial D_{\zeta}} \Phi(\zeta) d \zeta=\int_{\partial D_{\zeta}}\left(h_{1} x+h_{2} y\right) d \zeta=0 .
$$

The last equality is feasible if and only if $h_{2}=h_{1} e_{2}$.

Denote $U_{k}[\Phi(\zeta)]:=U_{k}(x, y)$, where $U_{k}(x, y), k=\overline{1,4}$, are components of the expansion (6). 
Our strategy is to find the functions $g_{1}$ and $g_{3}$ with using the method of indefinite coefficients and the boundary conditions (28) rewritten in the form of two equations with respect to sought-for functions $g_{1}, g_{3}$ and unknown hypercomplex numbers $h_{1}, h_{3}$ :

$$
\begin{gathered}
g_{k}(\zeta)+U_{k}\left[S_{\partial D_{\zeta}}\left[g_{1}\right](\zeta) e_{1}+S_{\partial D_{\zeta}}\left[g_{3}\right](\zeta) e_{2}\right]+ \\
+x U_{k}\left[h_{1}\right]+y U_{k}\left[h_{1} e_{2}\right]+U_{k}\left[h_{3}\right]=u_{k}(\zeta) \quad \forall \zeta \in \partial D_{\zeta}, k=1,3 .
\end{gathered}
$$

Using the equality (26), we obtain the following equalities:

$$
\begin{aligned}
& U_{1}\left[S_{\partial D_{\zeta}}\left[g_{1}\right](\zeta) e_{1}+S_{\partial D_{\zeta}}\left[g_{3}\right](\zeta) e_{2}\right]= \\
& =\frac{1}{2 \pi}\left(\operatorname{Im} \int_{\partial D} \frac{\widehat{g}_{1}(t)}{t^{2}} d t+\operatorname{Re} \int_{\partial D} \frac{\widehat{g}_{3}(t)}{t^{2}} d t\right)^{x+} \\
& +\frac{1}{2 \pi}\left(-\operatorname{Re} \int_{\partial D} \frac{\widehat{g}_{1}(t)}{t^{2}} d t+\operatorname{Im} \int_{\partial D} \frac{\widehat{g}_{1}(t)}{t^{3}} d t j^{y+}\right. \\
& +\frac{1}{2 \pi} \operatorname{Im} \int_{\partial D} \frac{\widehat{g}_{1}(t)}{t^{2}} d t+\frac{1}{2 \pi} \operatorname{Re} \int_{\partial D} \frac{\widehat{g}_{3}(t)}{t^{3}} d t, \\
& U_{3}\left[S_{\partial D_{\zeta}}\left[g_{1}\right](\zeta) e_{1}+S_{\partial D_{\zeta}}\left[g_{3}\right](\zeta) e_{2}\right]= \\
& =\frac{1}{2 \pi}\left(\operatorname{Re} \int_{\partial D} \frac{\widehat{g}_{1}(t)}{t^{2}} d t-\operatorname{Im} \int_{\partial D} \frac{\widehat{g}_{3}(t)}{t^{2}} d t\right)^{x+} \\
& +\frac{1}{2 \pi}\left(\operatorname{Im} \int_{\partial D} \frac{\widehat{g}_{1}(t)}{t^{2}} d t+\operatorname{Re} \int_{\partial D} \frac{\widehat{g}_{3}(t)}{t^{2}} d t\right)^{y+} \\
& +\frac{1}{2 \pi} \operatorname{Re} \int_{\partial D} \frac{\widehat{g}_{1}(t)}{t^{3}} d t-\frac{1}{2 \pi} \operatorname{Im} \int_{\partial D} \frac{\widehat{g}_{3}(t)}{t^{3}} d t .
\end{aligned}
$$

Now, it follows from the equalities $(34)-(36)$ that the functions $g_{1}, g_{3}$ can be expressed in the form

$$
g_{k}(\zeta)=u_{k}(\zeta)+a_{k, 1} x+a_{k, 2} y+a_{k, 0} \quad \forall \zeta \in \partial D_{\zeta}
$$

where unknown coefficients $a_{k, m}$ are real numbers for $k=1,3$ and $m=0,1,2$. 
Denote

$$
\begin{aligned}
A_{k} & :=\frac{1}{2 \pi} \operatorname{Re} \int_{\partial D} \frac{\widehat{u}_{k}(t)}{t^{2}} d t, \quad B_{k}:=\frac{1}{2 \pi} \operatorname{Im} \int_{\partial D} \frac{\widehat{u}_{k}(t)}{t^{2}} d t, \\
C_{k} & :=\frac{1}{2 \pi} \operatorname{Re} \int_{\partial D} \frac{\widehat{u}_{k}(t)}{t^{3}} d t, \quad D_{k}:=\frac{1}{2 \pi} \operatorname{Im} \int_{\partial D} \frac{\widehat{u}_{k}(t)}{t^{3}} d t, \quad k=1,3 .
\end{aligned}
$$

Substituting the expressions (37) into the equations (34) and taking into account the equalities

$$
\frac{1}{2 \pi} \int_{\partial D} \frac{x}{z^{2}} d z=\frac{i}{2}, \quad \frac{1}{2 \pi} \int_{\partial D} \frac{y}{z^{2}} d z=\frac{1}{2}, \quad \int_{\partial D} \frac{x}{z^{3}} d z=\int_{\partial D} \frac{y}{z^{3}} d z=0,
$$

$U_{1}\left[h_{1} e_{2}\right]=U_{3}\left[h_{1}\right], \quad U_{3}\left[h_{1} e_{2}\right]=U_{1}\left[h_{1}\right]-2 U_{4}\left[h_{1}\right]$, we obtain the relations

$$
\begin{aligned}
&\left(\frac{3}{2} a_{1,1}+\frac{1}{2} a_{3,2}+A_{3}+B_{1}+U_{1}\left[h_{1}\right]\right) x+ \\
&+\left(\frac{1}{2} a_{1,2}+\frac{1}{2} a_{3,1}-A_{1}+B_{3}+U_{3}\left[h_{1}\right]\right) y+ \\
&+a_{1,0}+C_{3}+D_{1}+U_{1}\left[h_{3}\right]=0 \\
&\left(\frac{1}{2} a_{1,2}+\frac{1}{2} a_{3,1}+A_{1}-B_{3}+U_{3}\left[h_{1}\right]\right) x+ \\
&+\left(\frac{1}{2} a_{1,1}+\frac{3}{2} a_{3,2}+A_{3}+B_{1}+U_{1}\left[h_{1}\right]-2 U_{4}\left[h_{1}\right]\right) y+ \\
& \quad+a_{3,0}+C_{1}-D_{3}+U_{3}\left[h_{3}\right]=0
\end{aligned}
$$

Consequently, we have a system of six equations with six real unknowns $a_{k, m}$ (with $k=1,3$ and $\left.m=0,1,2\right)$ and two hypercomplex parameters $h_{1}, h_{3}$ :

$$
\begin{aligned}
& a_{1,0}=-C_{3}-D_{1}-U_{1}\left[h_{3}\right], \\
& a_{3,0}=-C_{1}+D_{3}-U_{3}\left[h_{3}\right], \\
& a_{1,2}+a_{3,1}=2 A_{1}-2 B_{3}-2 U_{3}\left[h_{1}\right], \\
& a_{1,2}+a_{3,1}=-2 A_{1}+2 B_{3}-2 U_{3}\left[h_{1}\right], \\
& 3 a_{1,1}+a_{3,2}=-2 A_{3}-2 B_{1}-2 U_{1}\left[h_{1}\right], \\
& a_{1,1}+3 a_{3,2}=-2 A_{3}-2 B_{1}-2 U_{1}\left[h_{1}\right]+4 U_{4}\left[h_{1}\right] .
\end{aligned}
$$


It is obvious that this system is solvable if and only if $A_{1}-B_{3}=$ $=B_{3}-A_{1}$, i.e. $A_{1}=B_{3}$ that is equivalent to the condition (30). If this condition is satisfied, then the general solution of the system (38) contains an arbitrary real number $a_{1,2}$ and is of the form:

$$
\begin{aligned}
a_{1,0} & =-C_{3}-D_{1}-U_{1}\left[h_{3}\right], \\
a_{3,0} & =-C_{1}+D_{3}-U_{3}\left[h_{3}\right], \\
a_{3,1} & =-a_{1,2}-2 U_{3}\left[h_{1}\right], \\
a_{1,1} & =-\frac{1}{2} A_{3}-\frac{1}{2} B_{1}-\frac{1}{2} U_{1}\left[h_{1}\right]-\frac{1}{2} U_{4}\left[h_{1}\right], \\
a_{3,2} & =-\frac{1}{2} A_{3}-\frac{1}{2} B_{1}-\frac{1}{2} U_{1}\left[h_{1}\right]+\frac{3}{2} U_{4}\left[h_{1}\right] .
\end{aligned}
$$

Inasmuch as $h_{2}=h_{1} e_{2}$, the function

$$
\Phi(\zeta)=S_{D_{\zeta}}\left[g_{1}\right](\zeta) e_{1}+S_{D_{\zeta}}\left[g_{3}\right](\zeta) e_{2}+h_{1} \zeta+h_{3} \quad \forall \zeta \in D_{\zeta}
$$

have the boundary values (33) and is the general solution of (1-3)-problem for $D_{\zeta}$ in the class $\mathcal{M}$. In the formula (40) the functions $g_{1}, g_{3}$ are of the form (37), where the coefficients $a_{k, m}$ with $k=1,3$ and $m=0,1,2$ are determined by the equalities (39).

Finally, with using the equalities

$$
S_{D_{\zeta}}[1](\zeta)=e_{1}, 2 S_{D_{\zeta}}[x](\zeta)=\left(3 e_{1}+i e_{2}\right) \zeta, 2 S_{D_{\zeta}}[y](\zeta)=\left(-3 i e_{1}+e_{2}\right)
$$

for any $\zeta \in D_{\zeta}$, the formula (40) is reduced to the form (31).

Let $E:=\left\{(x, y) \in \mathbb{R}^{2}: x^{2}+y^{2}<1\right\}$ be the unit disk in the plane $\mathbb{R}^{2}$.

Consider the main biharmonic problem (see, for example, [16, p. 202]) on finding a biharmonic function $V: E \longrightarrow \mathbb{R}$ with given limiting values of its partial derivatives on the boundary $\partial E$ :

$$
\begin{aligned}
\lim _{(x, y) \rightarrow\left(x_{0}, y_{0}\right),(x, y) \in E} \frac{\partial V(x, y)}{\partial x} & =u_{1}\left(x_{0}, y_{0}\right), \\
\lim _{(x, y) \rightarrow\left(x_{0}, y_{0}\right),(x, y) \in E} \frac{\partial V(x, y)}{\partial y} & =u_{3}\left(x_{0}, y_{0}\right) \quad \forall\left(x_{0}, y_{0}\right) \in \partial E .
\end{aligned}
$$

It is shown in the paper [15] that this problem can be reduced to the (1-3)problem. Indeed, consider in the domain $D_{\zeta}$ a monogenic function

$$
\Phi_{1}(\zeta):=V(x, y) e_{1}+V_{2}(x, y) i e_{1}+V_{3}(x, y) e_{2}+V_{4}(x, y) i e_{2}
$$


for which $U_{1}\left[\Phi_{1}(\zeta)\right]=V(x, y)$. It follows from the Cauchy-Riemann condition (4) with $\Phi=\Phi_{1}$ that $\partial V_{3}(x, y) / \partial x=\partial V(x, y) / \partial y$. Therefore,

$$
\Phi_{1}^{\prime}(\zeta)=\frac{\partial V(x, y)}{\partial x} e_{1}+\frac{\partial V_{2}(x, y)}{\partial x} i e_{1}+\frac{\partial V(x, y)}{\partial y} e_{2}+\frac{\partial V_{4}(x, y)}{\partial x} i e_{2},
$$

and the main biharmonic problem with the boundary conditions (41) can be reduced to (1-3)-problem on finding a monogenic function $\Phi(\zeta):=\Phi_{1}^{\prime}(\zeta)$ in the domain $D_{\zeta}$.

Let us note that in this case the equality (30) is the well known necessary and sufficient condition for the solvability of the main biharmonic problem with the boundary conditions (41), see, for example, [16, p. 202].

Let us also note that the solvability of the (1-3)-problem is analogous to the solvability of Schwarz-type boundary value problems for polyanalytic functions and for analytic functions of several complex variables. In the paper [21] the Schwarz-type boundary value problem for the polyanalytic equation in a half-plane is solved in an explicit form without any complementary conditions. In the paper [22] Schwarz-type boundary value problems for analytic functions of several complex variables and for the inhomogeneous Cauchy-Riemann system in a polydisc are considered, ibid. solvability conditions for appropriate boundary value problems are obtained.

Results of this paper have been announced at a preprint of Arxiv (arXiv: 1202.0993v1 [math.CV]).

\section{References}

[1] V. F. Kovalev and I. P. Mel'nichenko, Biharmonic functions on the biharmonic plane, Reports Acad. Sci. USSR, ser. A., No. 8 (1981), 25-27 [in Russian].

[2] E. Study, Über systeme complexer zahlen und ihre anwendungen in der theorie der transformationsgruppen, Monatshefte für Mathematik, 1, No. 1 (1890), 283-354.

[3] I. P. Mel'nichenko, Biharmonic bases in algebras of the second rank, Ukr. Math. J., 38, No. 2 (1986), 224-226.

[4] E. Goursat, Cours d'analyse mathematique, Vol. 2, Gauthier-Villars, Paris, (1910). 
[5] Ju.Ju. Trokhimchuk, Continuous mappings and conditions of monogeneity, Israel Program for Scientific Translations, Jerusalem; Daniel Davey \& Co., Inc., New York, (1964).

[6] F. Brackx and R. Delanghe, Duality in hypercomplex functions theory, J. Funct. Anal., 37, No. 2 (1980), $164-181$.

[7] J. Ryan, Dirac operators, conformal transformations and aspects of classical harmonic analysis, J. Lie Theory, 8 (1998), $67-82$.

[8] A. Sudbery, Quaternionic analysis, Math. Proc. Camb. Phil. Soc., 85 (1979), $199-225$.

[9] V.V. Kravchenko and M.V. Shapiro, Integral representations for spatial models of mathematical physics, Pitman Research Notes in Mathematics, Addison Wesley Longman Inc. (1996).

[10] W. Sprössig, Quaternionic analysis and Maxwells equations, CUBO A Math. J., 7, no. 2 (2005), $57-67$.

[11] S. V. Gryshchuk and S. A. Plaksa, Monogenic functions in a biharmonic algebra, Ukr. Math. J., 61, No. 12 (2009), 1865-1876.

[12] S.A. Plaksa, Commutative Algebras Associated with Classic Equations of Mathematical Physics, In: Advances in Applied Analysis. Trends in Mathematics., Springer Basel AG (2012), 177-223.

[13] Plaksa S.A., Gryshchuk S.V., Shpakivskyi V.S., Commutative algebras of monogenic functions associated with classic equations of mathematical physic, in "Complex Analysis and Dynamical Systems IV", Contemporary Mathematics, 553 (2011), Amer. Math. Soc., Providence, RI, 245-258

[14] S. V. Gryshchuk and S. A. Plaksa, Monogenic functions in a biharmonic plane, Reports Acad. Sci. Ukraine, No. 12 (2009), 13-20 [in Russian].

[15] V. F. Kovalev, Biharmonic Schwarz problem, Preprint No. 86.16, Institute of Mathematics, Ukrainian Academy of Sciences, Kiev, (1986) [in Russian].

[16] Smirnov V.I., A Course of Higher Mathematics, Vol. 3, Part2, Pergamon Press, Oxford, (1964). 
[17] E. Hille and R. S. Phillips, Functional Analysis and Semi-Groups. Colloquium Publications 31, American Mathematical Society, Providence, (2000).

[18] O. F. Gerus, Some estimates of moduli of continuity of the Cauchy type integrals, Ukr. Math. J., 30, No. 5 (1978), 594-601.

[19] Gakhov F.D., Boundary Value Problems, Pergamon Press, Oxford (1966).

[20] S. A. Plaksa, Dirichlet problem for an axisymmetric potential in a simply connected domain of the meridian plane, Ukr. Math. J., 53, No. 12 (2001), 1976-1997.

[21] Begehr H., Chaudhary A., Kumar A., Bi-polyanalytic functions on the upper half plane, Complex Var. Elliptic Equ., 55 (2010), 305-316.

[22] Begehr H., Boundary value problems in $\mathbb{C}$ and $\mathbb{C}^{n}$, Acta Math. Viet., 22, No. 2 (1997), 407-425. 
